\title{
ON THE EXACT ASYMPTOTICS OF THE BUSY PERIOD IN GI/G/1 QUEUES
}

\author{
ZBIGNIEW PALMOWSKI, ${ }^{* *}$ Wrocław University and Utrecht University \\ TOMASZ ROLSKI, ${ }^{*}$ Wroctaw University
}

\begin{abstract}
In this paper we study the busy period in GI/G/1 work-conserving queues. We give the exact asymptotics of the tail distribution of the busy period under the light tail assumptions. We also study the workload process in the M/G/1 system conditioned to stay positive.

Keywords: GI/G/1 queue; busy period; workload process conditioned to stay positive; exponential change of measure; quasistationary distribution; exact asymptotics

2000 Mathematics Subject Classification: Primary 60K25
\end{abstract}

Secondary 60J25; 60J35

\section{Introduction}

The main object studied in this paper is the busy period, $\tau$, in single-server queues operating under a work-conserving discipline (e.g. first-come-first-served). Throughout the paper we assume that the traffic intensity, $\rho$, is less than 1, which implies that $\tau$ is finite almost surely, and that the initial workload is $x$. Recall that the busy period is greater than $t$ if and only if the workload process in the interval $[0, t]$ is strictly positive.

The case of the M/G/1 queue was considered by Kyprianou (1971), who studied the asymptotics of the tail probability of the busy period where a service time has a meromorphic moment generating function $\hat{m}_{B}(\theta)$. Let $\lambda$ be the arrival rate and let $\kappa(s)=\lambda\left(\hat{m}_{B}(s)-1\right)-s$. Kyprianou (1971) considered the light tail case, namely that there exists a $\theta_{0}>0$ such that $\kappa^{\prime}\left(\theta_{0}\right)=0$ or, equivalently, $\lambda \hat{m}_{B}^{\prime}\left(\theta_{0}\right)=1$. Using analytical methods he proved that

$$
\mathrm{P}_{x}(\tau>t) \sim h(x) \mathrm{e}^{-\gamma t} t^{-3 / 2}
$$

as $t \rightarrow \infty$, where $h(x)=C x \mathrm{e}^{\theta_{0} x}$,

$$
\gamma=-\kappa\left(\theta_{0}\right), \quad C=\frac{1}{-\kappa\left(\theta_{0}\right) \sigma \sqrt{2 \pi}}, \quad \sigma^{2}=\lambda \hat{m}_{B}^{\prime \prime}\left(\theta_{0}\right) .
$$

A result of this type for the density function was first given in the appendix of the book of Cox and Smith (1961) (Equation (46) on page 154), and it was proved using the saddle-point method.

The main result of this paper is the exact asymptotics for the tail of the distribution function of the busy period in the GI/G/1 queue. Of course, in this case we must first markovize the workload process by attaching the second component of the remaining arrival time process to obtain a Markovian setting; therefore, in Theorem 2.1 we give the asymptotics of $\mathrm{P}_{(x, z)}(\tau>t)$, where $x$ and $z$ are the initial workload and remaining arrival time, respectively. In Corollary 2.1 we state

Received 24 September 2004; revision received 13 July 2006.

* Postal address: Mathematical Institute, Wrocław University, pl. Grunwaldzki 2/4, 50-384 Wrocław, Poland.

**Email address: zpalma@math.uni.wroc.pl 
a result similar to (1.1) for GI/G/1 a work-conserving system. Recently, a weaker logarithmic asymptotics was found in GI/G/1 queues by Mandjes and Zwart (2006), as a by-product of their studies of GI/G/1 processor-sharing queues.

In the light of Kyprianou's (1971) studies, a naturally related concept is the workload process conditioned to stay positive. This notion is a counterpart of ones such as the random walk conditioned to stay positive (see, e.g. Keener (1992) and Bertoin and Doney (1994)) or the Brownian motion conditioned to stay positive derived by Knight (1969). In Section 4 we study this concept for an M/G/1 queue, completing Kyprianou's (1971) studies.

\section{Main result}

We now introduce the queueing notation used throughout the paper. Customers arrive at the instants $T_{0}, T_{0}+T_{1}, T_{1}+T_{2}, \ldots$, where $T_{0}, T_{1}, \ldots$ are the interarrival times. If there is an arrival at 0 then we set $T_{0}=0$. The customer arriving at $\varrho_{n}:=T_{0}+\cdots+T_{n}$ has associated service time $B_{n}$. Let $S(t)=\sum_{j=1}^{N(t)} B_{j}$ be the cumulative service time up to time $t$, where $N(t)$ denotes the number of all arrivals by time $t$ (including the one at time 0 if there is one). That is,

$$
N(t)=\sum_{j=0}^{\infty} \mathbf{1}\left(T_{0}+\cdots+T_{n} \leq t\right) .
$$

We assume that the random variables $T_{0}, T_{1}, T_{2}, \ldots$ are independent and that $T_{1}, T_{2}, T_{3}, \ldots$ are independent, identically distributed random variables. This sequence of variables is furthermore independent of an independent, identically distributed sequence $B_{1}, B_{2}, \ldots$ with the nonlattice distribution $F_{B}$. Throughout the paper we also assume that a generic $T=T_{1}$ and $B=B_{1}$ are light tailed, that is, there exists a $\delta>0$ such that

$$
\mathrm{E}_{x} \mathrm{e}^{\delta T}<\infty, \quad \mathrm{E}_{x} \mathrm{e}^{\delta B}<\infty .
$$

Under $\mathrm{P}_{x}$ we define the process $X(t)=x+S(t)-t$. Let $\mathrm{E}_{x}$ be a corresponding expectation. For all systems working under a work-conserving discipline the work load process is

$$
V(t)=\max _{0 \leq s \leq t}(X(t), X(t)-X(s)) .
$$

By the busy period we mean the following stopping time:

$$
\tau=\min \{t: V(t)=0\}=\min \{t: x+S(t)-t=0\} .
$$

Our permanent assumption is that $\tau$ is a proper random variable, that is, $\rho=\mathrm{E}_{x} B / \mathrm{E}_{x} T<1$. Note that the two processes $X(t)$ and $V(t)$ are identical within the first busy period. We let

$$
\lambda=\lim _{t \rightarrow \infty} \frac{N(t)}{t} \quad \text { almost surely. }
$$

With the above queueing description in continuous time we associate the following random walk. For $\zeta_{i}=B_{i}-T_{i}(i=1,2, \ldots)$, let

$$
Z_{0}=x, \quad Z_{n}=\sum_{k=1}^{n} \zeta_{k}, \quad n=1,2, \ldots,
$$

and $v=\min \left\{n: Z_{n}<0\right\}$. 
Note that $\mathrm{E}_{x} \zeta<0$ since $\rho<1$. We also consider a Palm version of the problem with probability measure $\mathrm{P}_{x}^{\circ}$, under which $T_{0}=0$.

For a random variable $Y$ we denote by $F_{Y}(\cdot)$ its distribution and by $\hat{m}_{Y}(s)=\operatorname{E} \exp (s Y)$ its moment generating function. Also, we use the notation $\hat{l}_{\xi}(s)=\int_{0}^{\infty} \mathrm{e}^{-s x} \xi(\mathrm{d} x)$ for the Laplace transform of a measure $\xi$ which is not necessarily finite. We will write $k(t) \sim l(t)$ to mean that $\lim _{t \rightarrow \infty} k(t) / l(t)=1$.

Let $\theta_{\mathrm{r}}=\sup \left\{\theta: \hat{m}_{B}(\theta)<\infty\right\}>0$. The function $\kappa(\theta)$ is the unique solution to

$$
\hat{m}_{B}(\theta) \hat{m}_{T}(-\kappa(\theta)-\theta)=1
$$

in the interval $\left(-\infty, \theta_{\mathrm{r}}\right)$. The function $\kappa(\theta)$ is convex and $\kappa^{\prime}(0)=\rho-1$ (see Rolski et al. (1999, Lemma 11.5.1)). We assume that there exists a $\theta_{0}<\theta_{\mathrm{r}}$ such that $\kappa^{\prime}\left(\theta_{0}\right)=0$. If the above condition, together with (2.1), is fulfilled, then we say that the light tail case pertains.

Unfortunately, neither $V(t)$ nor $X(t)$ is a Markovian process unless the queue is of M/G/1 type. However, the process $(X(t), Z(t))$ is Markovian, where the process

$$
Z(t)=\sum_{j=0}^{N(t)} T_{j}-t
$$

measures the remaining time to the next arrival. We also set the initial conditions $X(0)=x$ and $Z(0)=z$, which will be indicated to hold by the notation $\mathrm{P}_{(x, z)}$.

Actually, we consider the Markov process $(X(t), Z(t), t)$, which falls into the framework of piecewise-deterministic Markov processes; for the theory, see Davis (1993, Chapter II). The full generator has the form

$$
\mathcal{A} f(x, z, t)=\frac{\partial}{\partial t} f(x, z, t)-\frac{\partial}{\partial x} f(x, z, t)-\frac{\partial}{\partial z} f(x, z, t),
$$

where $f$ is an absolutely continuous function fulfilling the boundary condition

$$
f(x, 0, t)=\int_{0}^{\infty} \int_{0}^{\infty} f(x+y, z, t) \mathrm{d} F_{B}(y) \mathrm{d} F_{T}(z)
$$

and such that

$$
\mathrm{E}\left(\sum_{i \leq t}\left|f\left(X\left(\tau \wedge \varrho_{i}\right)\right)-f\left(X\left(\tau \wedge \varrho_{i}-\right)\right)\right|\right)<\infty
$$

for each $t \geq 0$.

We first show how to remove the drift. Let $\theta<\theta_{\mathrm{r}}$. Consider a function $g(x, v, t)=$ $k(z) \mathrm{e}^{\theta x} \mathrm{e}^{-\kappa(\theta) t}$. For $g$ to be a harmonic function, we have to check that $\mathcal{A} g=0$ and that the conditions (2.4) and (2.5) hold. This means that $k(v)$ solves $-\kappa(\theta) k(z)-\theta k(z)-k^{\prime}(z)=0$ and, hence, that $k(z)=k(0) \mathrm{e}^{-(\kappa(\theta)+\theta) z}$. We also find that the boundary condition (2.4) holds, because $\kappa(\theta)$ is the solution to (2.3). Since $\theta<\theta_{\mathrm{r}}$, we can show (2.5) following Rolski et al. (1999, Chapter 11.3.1). Note that $\kappa^{\prime}(0)=\rho-1<0$. Thus, $g(x, z, t)=\mathrm{e}^{\theta x-(\kappa(\theta)+\theta) z} \mathrm{e}^{-\kappa(\theta) t}$ and the process

$$
\mathcal{E}(t)=\mathrm{e}^{-\theta x} \mathrm{e}^{(\theta+\kappa(\theta)) z} \mathrm{e}^{\theta X(t)} \mathrm{e}^{-(\theta+\kappa(\theta)) Z(t)} \mathrm{e}^{-\kappa(\theta) t}
$$

is a mean-1 exponential martingale defined by the function $g$.

We now introduce the new probability measure $\mathrm{P}_{(x, z) ; \theta}$, defined by

$$
\mathrm{dP}_{(x, z) ; \theta \mid t}=\mathscr{E}(t) \mathrm{dP}_{(x, y) \mid t}, \quad t \geq 0,
$$


where the subscript ' $\mid t$ ' denotes the restriction of a probability measure to $\mathcal{F}_{t}^{X, Z}$, the $\sigma$-field generated by the process $(X(t), Z(t), t)$ up to time $t$. From Theorem 5.3 of Palmowski and Rolski (2002), under the new probability we find that the distributions of $T$ and $B$ are

$$
\begin{aligned}
\mathrm{d} F_{T}^{\theta}(v) & =\frac{1}{\hat{m}_{T}(-\kappa(\theta)-\theta)} \mathrm{e}^{-(\kappa(\theta)+\theta) v} \mathrm{~d} F_{T}(v), \\
\mathrm{d} F_{B}^{\theta}(v) & =\frac{1}{\hat{m}_{B}(\theta)} \mathrm{e}^{-\theta v} \mathrm{~d} F_{B}(v),
\end{aligned}
$$

and, so,

$$
\mathrm{E}_{(x, z) ; \theta} T=\frac{\hat{m}_{T}^{\prime}(-\kappa(\theta)-\theta)}{\hat{m}_{T}(-\kappa(\theta)-\theta)}, \quad \mathrm{E}_{(x, z) ; \theta} B=\frac{\hat{m}_{B}^{\prime}(\theta)}{\hat{m}_{B}(\theta)} .
$$

Taking the first derivative with respect to $\theta$ in (2.3), we obtain

$$
\hat{m}_{B}^{\prime}(\theta) \hat{m}_{T}(-\kappa(\theta)-\theta)-\hat{m}_{B}(\theta) \hat{m}_{T}^{\prime}(-\kappa(\theta)-\theta)-\kappa^{\prime}(\theta) \hat{m}_{B}(\theta) \hat{m}_{T}^{\prime}(-\kappa(\theta)-\theta)=0 .
$$

Using (2.7) and (2.8) we can prove that the workload process has zero drift under $\mathrm{P}_{(x, z) ; \theta_{0}}$.

Lemma 2.1. Under the new probability, the drift,

$$
\mathrm{E}_{(x, z) ; \theta} B-\mathrm{E}_{(x, z) ; \theta} T=\frac{\hat{m}_{B}^{\prime}(\theta) \hat{m}_{T}(-\kappa(\theta)-\theta)-\hat{m}_{B}(\theta) \hat{m}_{T}^{\prime}(-\kappa(\theta)-\theta)}{\hat{m}_{B}(\theta) \hat{m}_{T}(-\kappa(\theta)-\theta)},
$$

is 0 if and only if $\kappa^{\prime}(\theta)=0$.

Let $\beta=-\left(\theta_{0}+\kappa\left(\theta_{0}\right)\right)$, which is negative by (2.3). Under the new probability $\tilde{\mathrm{P}}_{x}$, consider the random walk $Z_{n}$ such that the distribution of the generic increment $\zeta$ is

$$
\tilde{\mathrm{P}}_{x}(\zeta \in \Lambda)=r^{-1} \mathrm{E}_{x ; \theta_{0}}^{\circ}\left(\mathrm{e}^{\beta \zeta} ; \zeta \in \Lambda\right),
$$

where $r=\mathrm{E}_{x ; \theta_{0}}^{\circ} \mathrm{e}_{\tilde{\mathrm{E}}}^{\beta \zeta}$ and $\Lambda$ is a Borel set. Note that $\tilde{\mathrm{E}}_{x} \zeta<0$, since $\mathrm{E}_{x ; \theta_{0}}^{\circ} T \mathrm{e}^{-\beta T}>\mathrm{E}_{x ; \theta_{0}}^{\circ} B \mathrm{e}^{\beta B}$. Let $\tilde{\kappa}(\theta)=\log \tilde{\mathrm{E}}_{x} \mathrm{e}^{\theta \zeta}$ and $\kappa_{\theta_{0}}(\theta)=\log \mathrm{E}_{x ; \theta_{0}} \mathrm{e}^{\theta \zeta}$. Then $\tilde{\kappa}(\theta)=\kappa_{\theta_{0}}(\theta+\beta)-\kappa_{\theta_{0}}(\beta)$ and $\tilde{\kappa}^{\prime}(-\beta)=\kappa_{\theta_{0}}^{\prime}(0)=0$. Moreover,

$$
r=\mathrm{e}^{\kappa \theta_{0}(\beta)}=\mathrm{e}^{-\tilde{\kappa}(-\beta)}>1 .
$$

Let

$$
H(x)=D \sum_{n=0}^{\infty} \mathrm{P}_{x ; \theta_{0}}^{\circ}\left(L_{n}=n, Z_{n} \geq 0\right)
$$

where

$$
L_{n}=\min \left\{r \geq 0: Z_{r}=\min _{k \leq n} Z_{k}\right\}
$$

is a decreasing ladder epoch and

$$
D=\frac{1}{-\beta \sqrt{2 \pi \kappa_{\theta_{0}}^{\prime \prime}(0)}} \frac{\tilde{\mathrm{E}}\left(r^{\nu}-1\right)}{r-1} .
$$

Let $\lambda(\theta)=\left(\mathrm{E}_{x ; \theta} T\right)^{-1}$. We can now state the main result, whose proof will be presented in Section 3 . 
Theorem 2.1. For all $x$ and $z, x \geq z \geq 0$, as $t \rightarrow \infty$,

$$
\mathrm{P}_{(x, z)}(\tau>t) \sim \frac{r-1}{-\kappa\left(\theta_{0}\right)} \mathrm{e}^{\theta_{0} x} \mathrm{e}^{-\left(\theta_{0}+\kappa\left(\theta_{0}\right)\right) z} H(x-z) \mathrm{e}^{\kappa\left(\theta_{0}\right) t} \frac{1}{\sqrt{\lambda\left(\theta_{0}\right)} t^{3 / 2}} .
$$

In the following corollary of Theorem 2.1 we write $\mathrm{P}_{x}(\cdot)$ for $\mathrm{P}_{x, z}(\cdot) \mathrm{d} F_{Z(0)}(z)$.

Corollary 2.1. For all $x>0$,

$$
\mathrm{P}_{x}(\tau>t) \sim h(x) \mathrm{e}^{\kappa\left(\theta_{0}\right) t} t^{-3 / 2}
$$

as $t \rightarrow \infty$, where

$$
h(x)=\frac{r-1}{-\kappa\left(\theta_{0}\right)} \frac{1}{\sqrt{\lambda\left(\theta_{0}\right)}} \mathrm{e}^{\theta_{0} x} \int_{0}^{x} \mathrm{e}^{-\left(\theta_{0}+\kappa\left(\theta_{0}\right)\right) z} H(x-z) \mathrm{d} F_{Z(0)}(z) .
$$

We complete the section by comparing (2.12) with (1.1) for the M/M/1 queue. In this case $F_{Z(0)}(\cdot)$ is exponentially distributed with parameter $\lambda$. The following argument demonstrates that indeed $h(x)=C \mathrm{e}^{\theta_{0} x} x$, where $C$ is as defined in (1.2). Suppose that $B \stackrel{\mathrm{D}}{=} \operatorname{Exp}(\varphi)$ and $T \stackrel{\mathrm{D}}{=} \operatorname{Exp}(\lambda)$, where $\stackrel{\mathrm{D}}{=}$, denotes equality in distribution. Then $\theta_{0}=\sqrt{\varphi}(\sqrt{\varphi}-\sqrt{\lambda})$ and $\kappa\left(\theta_{0}\right)=\varphi-\lambda-2 \theta_{0}$. Hence, we compute that $\beta=-\left(\theta_{0}+\kappa\left(\theta_{0}\right)\right)=\sqrt{\lambda}(\sqrt{\lambda}-\sqrt{\varphi})$. Note that $\lambda-\beta=\sqrt{\varphi \lambda}$. Moreover, we have $B \stackrel{\mathrm{D}}{=} T \stackrel{\mathrm{D}}{=} \operatorname{Exp}(\sqrt{\varphi \lambda})$ under $\mathrm{P}_{x ; \theta_{0}}$. Thus, from Feller $(1966$, p. 387), we obtain $H(x)=C(1+\sqrt{\varphi \lambda} x)$ because under $\mathrm{P}_{x ; \theta_{0}}$ the downward ladder epoch is exponentially distributed with parameter $\sqrt{\lambda \varphi}$. Straightforward calculations now show that

$$
\int_{0}^{x} \mathrm{e}^{-\left(\theta_{0}+\kappa\left(\theta_{0}\right)\right) z} H(x-z) \mathrm{d} F_{Z(0)}(z)=\lambda \int_{0}^{x} \mathrm{e}^{-(\lambda-\beta) z} H(x-z) \mathrm{d} z=D \lambda x,
$$

where $D$ is the constant given in (2.11). Thus, it suffices to demonstrate that

$$
\frac{r-1}{-\kappa\left(\theta_{0}\right)} \frac{1}{\sqrt{\lambda\left(\theta_{0}\right)}} \lambda D=C
$$

which is equivalent to

$$
\frac{\tilde{\mathrm{E}}\left(r^{\nu}-1\right)}{-\beta \sqrt{\kappa_{\theta_{0}}^{\prime \prime}(0)}} \frac{\lambda}{\sqrt{\lambda\left(\theta_{0}\right)}}=\frac{1}{\sigma}
$$

where $\sigma$ is as defined in (1.2). Now (2.6) yields

$$
\operatorname{var}_{x ; \theta_{0}} S(1)=\lambda\left(\theta_{0}\right) \mathrm{E}_{\theta_{0}} B^{2}=\lambda \hat{m}_{B}\left(\theta_{0}\right) \frac{\hat{m}_{B}^{\prime \prime}\left(\theta_{0}\right)}{\hat{m}_{B}\left(\theta_{0}\right)}=\sigma^{2},
$$

and, hence, $\sigma^{2}=2 \lambda\left(\theta_{0}\right) / \lambda \varphi$. Moreover, from (2.9) we obtain

$$
\begin{aligned}
\tilde{\mathrm{E}} r^{v} & =\sum_{n=1}^{\infty} r^{n} \tilde{\mathrm{P}}(v=n)=\sum_{n=1}^{\infty} r^{n} r^{-n} \mathrm{P}_{\theta_{0}}^{\circ}\left(\mathrm{e}^{\beta Z_{n}} ; v=n\right)=\mathrm{E}_{\theta_{0}}^{\circ} \mathrm{e}^{\beta Z_{v}} \\
& =\sqrt{\lambda \varphi} \int_{0}^{\infty} \mathrm{e}^{-\beta x} \mathrm{e}^{-\sqrt{\lambda \varphi} x} \mathrm{~d} x=\frac{\sqrt{\lambda \varphi}}{\sqrt{\lambda \varphi}+\beta}=\sqrt{\frac{\varphi}{\lambda}} .
\end{aligned}
$$

By inspection we see that $\kappa_{\theta_{0}}^{\prime \prime}(0)=2 / \lambda \varphi$. This completes the proof of (2.13). 


\section{Proof of Theorem 2.1}

We will use the following properties of a class of regularly varying discrete sequences. The proofs can be found in Chover et al. (1973).

Lemma 3.1. Let $\left(a_{n}^{(i)}\right), i=1,2,3$, be sequences of nonnegative numbers and let $A_{i}=$ $\sum_{n=1}^{\infty} a_{n}^{(i)}, i=2$, 3. If $a_{n}^{(1)} \sim a_{1} n^{-1 / 2}, a_{n}^{(2)} \sim a_{2} n^{-3 / 2}$, and $a_{n}^{(3)} \sim a_{3} n^{-3 / 2}$, then

$$
\left(a^{(1)} * a^{(3)}\right)_{n} \sim a_{1} A_{3} n^{-1 / 2} \text { and }\left(a^{(2)} * a^{(3)}\right)_{n} \sim\left(a_{2} A_{3}+a_{3} A_{2}\right) n^{-3 / 2},
$$

where '*' denotes convolution.

The more detailed analysis that can be found in Doney (1989) and Iglehart (1974) allows us to prove the following lemma.

Lemma 3.2. For some function $c(x)$,

$$
\tilde{\mathrm{P}}_{x}(v>n)=H(x) r^{-n} n^{-3 / 2}\left(1+\frac{c(x)}{n}+o\left(n^{-1}\right)\right)
$$

in the limit as $n \rightarrow \infty$.

Proof. Let $S_{n}=-\left(Z_{n}-x\right)$, where $Z_{n}$ is as defined in (2.2), be a random walk. It starts from 0 and drifts to $\infty$. Let $M_{n}=\max _{k \leq n} S_{k}$. To prove the lemma we have to generalize Theorem II of Doney (1989) (see also Theorem 2.1 of Iglehart (1974)). Basically, we need to generalize Lemma 4(ii) of Doney (1989) and prove that

$$
\begin{aligned}
a_{n}(t) & =\frac{1}{n}\left[\mathrm{E}\left(\mathrm{e}^{-t S_{n}} ; S_{n}>0\right)+\mathrm{P}\left(S_{n} \leq 0\right)\right] \\
& =\frac{1}{-\beta \sqrt{2 \pi \tilde{\kappa}^{\prime \prime}(-\beta)}} \frac{t}{t-\beta} \mathrm{e}^{\kappa\left(\theta_{0}\right) n} n^{-3 / 2}\left[1+\frac{Q(t)}{n}+o\left(n^{-1}\right)\right]
\end{aligned}
$$

for some function $Q(t)$. We start from Spitzer's identity,

$$
\sum_{n=0}^{\infty} s^{n} \mathrm{E}\left(\mathrm{e}^{-t M_{n}}\right)=\exp \left(\sum_{n=1}^{\infty} s^{n} a_{n}(t)\right) .
$$

Using Lemma 3.1 and following ideas from the proof of Theorem 2.1 of Iglehart (1974) (see also Lemma 1 of Doney (1989)), in the next step we obtain

$$
\begin{aligned}
& \mathrm{E}\left(\mathrm{e}^{-t M_{n}}\right) \\
& \quad=\exp \left(\sum_{n=1}^{\infty} \mathrm{e}^{-\kappa\left(\theta_{0}\right) n} a_{n}(t)\right) \frac{1}{-\beta \sqrt{2 \pi \tilde{\kappa}^{\prime \prime}(-\beta)}} \frac{t}{t-\beta} \mathrm{e}^{\kappa\left(\theta_{0}\right) n} n^{-3 / 2}\left[1+\frac{Q_{1}(t)}{n}+o\left(n^{-1}\right)\right],
\end{aligned}
$$

where $Q_{1}(t)$ is a certain function. Then, as in the proof of Theorem II of Doney (1989), we can recover (3.1) with a function $c(x)$ of a form uninteresting to us and

$$
H(x)=D \mathrm{e}^{-\beta x} \sum_{n=0}^{\infty} r^{n} \tilde{\mathrm{E}}_{0}\left(\mathrm{e}^{\beta S_{n}} ; L_{n}^{S}=n, 0 \leq S_{n} \leq x\right),
$$


where $L_{n}^{S}$ are increasing ladder epochs of the random walk $S_{n}$. In calculating the constant $D$ we use the identity $\tilde{\kappa}^{\prime \prime}(-\beta)=\kappa_{\theta_{0}}^{\prime \prime}(0)$. The function $H(x)$ given in (3.3) can, after some work, be transformed into

$$
H(x)=D \sum_{n=0}^{\infty} r^{n} \tilde{\mathrm{E}}_{x}\left(\mathrm{e}^{-\beta Z_{n}} ; L_{n}=n, Z_{n} \geq 0\right) .
$$

Next, using (2.9) we can write the function in the form in (2.10). Finally, to demonstrate (3.2) we follow the proof of Lemma 4(ii) of Doney (1989). However, we use the asymptotics

$$
\mathrm{P}\left(S_{n} \leq x\right)=\frac{1}{\theta_{1} \sqrt{2 \pi \tilde{\kappa}^{\prime \prime}\left(\theta_{1}\right)}} \mathrm{e}^{x \theta_{1}} \mathrm{e}^{\kappa\left(\theta_{1}\right) n} n^{-1 / 2}\left(1+d(x) \frac{1}{n}+o\left(n^{-1}\right)\right),
$$

which holds uniformly in $x \leq \frac{1}{2} n \mathrm{E} S_{1}$, instead of Equation (2.8) of Doney (1989). Here $\theta_{1}$ is a solution to $\kappa^{\prime}(\theta)=x / n$ and $d(x)$ is a certain function. The proof of (3.4) follows from the proof of Theorem 1 of Petrov (1965) using Theorem VI.3.4 of Petrov (1972) instead of Equation (4.12) therein. This completes the proof of the lemma.

Since, under the Palm probability measure, we have $Z(0)=0$, we may write $\mathrm{P}_{(x, 0) ; \theta_{0}}^{\circ}=$ $\mathrm{P}_{x ; \theta_{0}}^{\circ}$. Recall that under $\mathrm{P}_{x ; \theta_{0}}^{\circ}$ the random walk $Z_{n}$ defined in (2.2) oscillates. We have

$$
\tau=\inf \{s \geq 0: X(s)<0\}>t
$$

if and only if $v>N(t)$, where $N(t)$ is the number of customers that have arrived up to time $t$. By the law of large numbers, $\lim _{t \rightarrow \infty} N(t) / t=\lambda\left(\theta_{0}\right) \mathrm{P}_{x ; \theta_{0}}^{\circ}$-almost surely. Furthermore, by the central limit theorem (see, e.g. Theorem IX.4.28 of Petrov (1972)), for each $\delta>0$,

$$
\mathrm{P}_{x ; \theta}^{\circ}\left(\left|\frac{N(t)}{t}-\lambda\left(\theta_{0}\right)\right|>\delta\right)=o\left(t^{-3 / 2}\right)
$$

since $\mathrm{Ee}^{\varsigma T}<\infty$ for some $\varsigma>0$. Note also that $Z(\tau)=-Z_{v}$.

Lemma 3.3. For $c_{1}=r /(2(r-1))$, as $t \rightarrow \infty$ we have

$$
\begin{aligned}
\mathrm{E}_{x ; \theta_{0}}^{\circ}\left(\mathrm{e}^{-\beta Z(\tau)} ; \tau>t\right) & =\mathrm{E}_{x ; \theta_{0}}^{\circ}\left(\mathrm{e}^{\beta Z_{v}} ; v>\lambda\left(\theta_{0}\right) t\right)+o\left(t^{-3 / 2}\right) \\
& =\frac{2 H(x)(r-1)}{\sqrt{\lambda\left(\theta_{0}\right) t}}\left(1+c_{1}\left(\lambda\left(\theta_{0}\right) t\right)^{-1}+o\left(t^{-1}\right)\right) .
\end{aligned}
$$

Proof. We prove the first asymptotic equivalence. For the upper bound we have the estimation

$$
\begin{aligned}
\mathrm{E}_{x ; \theta_{0}}^{\circ}\left(\mathrm{e}^{-\beta Z(\tau)} ; \tau>t\right) & \leq \mathrm{E}_{x ; \theta_{0}}^{\circ}\left(\mathrm{e}^{-\beta Z(\tau)} ; \tau>t ;\left|\frac{N(t)}{t}-\lambda\left(\theta_{0}\right)\right|<\delta\right)+o\left(t^{-3 / 2}\right) \\
& \leq \mathrm{E}_{x ; \theta_{0}}^{\circ}\left(\mathrm{e}^{\beta Z_{v}} ; v>\left(\lambda\left(\theta_{0}\right)-\delta\right) t\right)+o\left(t^{-3 / 2}\right) .
\end{aligned}
$$

For the lower bound, we have

$$
\begin{aligned}
\mathrm{E}_{x ; \theta_{0}}^{\circ}\left(\mathrm{e}^{-\beta Z(\tau)} ; \tau>t\right) & \geq \mathrm{E}_{x ; \theta_{0}}^{\circ}\left(\mathrm{e}^{-\beta Z(\tau)} ; \tau>t ;\left|\frac{N(t)}{t}-\lambda\left(\theta_{0}\right)\right|<\delta\right)-o\left(t^{-3 / 2}\right) \\
& \geq \mathrm{E}_{x ; \theta_{0}}^{\circ}\left(\mathrm{e}^{\beta Z_{v}} ; v>\left(\lambda\left(\theta_{0}\right)+\delta\right) t\right)-o\left(t^{-3 / 2}\right) .
\end{aligned}
$$


Letting $\delta \rightarrow 0$ completes the proof of the first part. To prove the second asymptotic equivalence, note that

$$
\mathrm{E}_{x ; \theta_{0}}^{\circ}\left(\mathrm{e}^{\beta Z_{v}} ; v>n\right)=\sum_{k>n} \mathrm{E}_{x ; \theta_{0}}^{\circ}\left(\mathrm{e}^{\beta Z_{k}} ; v=k\right)=\sum_{k>n} r^{k} \tilde{\mathrm{P}}_{x}(v=k)=\tilde{\mathrm{E}}_{x}\left(r^{v} ; v>n\right) .
$$

From $\tilde{\mathrm{P}}_{x}(v>n)=\sum_{k>n} \tilde{\mathrm{P}}_{x}(v=k)$ and Lemma 3.2, we conclude that

$$
\tilde{\mathrm{P}}_{x}(v=n)=(r-1) H(x) r^{-n} n^{-3 / 2}\left(1+c_{0} n^{-1}+o\left(n^{-1}\right)\right)
$$

for $c_{0}=3 r /(2(r-1))$. Similarly, since $\tilde{\mathrm{E}}_{x}\left(r^{\nu} ; v>n\right)=\sum_{k>n} r^{k} \tilde{\mathrm{P}}_{x}(v=k)$, we obtain

$$
\tilde{\mathrm{E}}_{x}\left(r^{\nu} ; v>n\right)=2(r-1) H(x) n^{-1 / 2}\left(1+c_{1} n^{-1}+o\left(n^{-1}\right)\right) .
$$

This completes the proof.

Lemma 3.4. We have

$$
\begin{aligned}
\mathrm{P}_{(x, z)}(\tau>t)= & -\kappa\left(\theta_{0}\right) \mathrm{e}^{\theta_{0} x} \mathrm{e}^{-\left(\theta_{0}+\kappa\left(\theta_{0}\right)\right) z} \mathrm{e}^{\kappa\left(\theta_{0}\right) t} \\
& \times \int_{0}^{\infty} \mathrm{e}^{\kappa\left(\theta_{0}\right) v}\left[\mathrm{E}_{(x, z) ; \theta_{0}}\left(\mathrm{e}^{\left(\theta_{0}+\kappa\left(\theta_{0}\right)\right) Z(\tau)} ; \tau>t\right)\right. \\
& \left.\quad-\mathrm{E}_{(x, z) ; \theta_{0}}\left(\mathrm{e}^{\left(\theta_{0}+\kappa\left(\theta_{0}\right)\right) Z(\tau)} ; \tau>t+v\right)\right] \mathrm{d} v .
\end{aligned}
$$

Proof. Standard arguments based on the likelihood ratio identity as in Lemma 10.2.2 of Rolski et al. (1999) (see also the section on the change of measure in Asmussen (2003, Chapter XIII.3)) yield

$$
\mathrm{P}_{(x, z)}(\tau>t)=\mathrm{e}^{\theta_{0} x} \mathrm{e}^{-\left(\theta_{0}+\kappa\left(\theta_{0}\right)\right) z} \mathrm{E}_{(x, z) ; \theta_{0}}\left(\mathrm{e}^{\left(\theta_{0}+\kappa\left(\theta_{0}\right)\right) Z(\tau)} \mathrm{e}^{\kappa\left(\theta_{0}\right) \tau} ; \tau>t\right) .
$$

Integrating by parts completes the proof.

From Lemma 3.4 we immediately obtain a Lundberg-type bound for $\mathrm{P}_{(x, z)}(\tau>t)$.

Corollary 3.1. For all $t \geq 0, \mathrm{P}_{(x, z)}(\tau>t) \leq \mathrm{e}^{\theta_{0} x} \mathrm{e}^{-\left(\theta_{0}+\kappa\left(\theta_{0}\right)\right) z} \mathrm{e}^{\kappa\left(\theta_{0}\right) t}$.

Proof. Rewrite (3.5) in the form

$$
\begin{aligned}
\mathrm{P}_{(x, z)}(\tau>t)= & -\kappa\left(\theta_{0}\right) \mathrm{e}^{\theta_{0} x} \mathrm{e}^{-\left(\theta_{0}+\kappa\left(\theta_{0}\right)\right) z} \mathrm{e}^{\kappa\left(\theta_{0}\right) t} \\
& \times \int_{0}^{\infty} \mathrm{e}^{\kappa\left(\theta_{0}\right) v} \mathrm{E}_{(x, z) ; \theta_{0}}\left(\mathrm{e}^{\left(\theta_{0}+\kappa\left(\theta_{0}\right)\right) Z(\tau)} ; t+v>\tau>t\right) \mathrm{d} v .
\end{aligned}
$$

From this, since $\theta_{0}+\kappa\left(\theta_{0}\right)>0$ and $Z(\tau) \leq 0$, the inequality follows.

In the proof of the main result we will also use the following technical lemma.

Lemma 3.5. If $f(t)=A^{\prime} t^{-1 / 2}+B t^{-3 / 2}+o\left(t^{-3 / 2}\right)$, for some $A^{\prime} \neq 0$, then

$$
f(t+v)-f(t)=-v \frac{A^{\prime}}{2} t^{-3 / 2}+o\left(t^{-3 / 2}\right) .
$$

Proof. Since $(1+s)^{\alpha}=1+\alpha s+o(s)$, we have

$$
\begin{aligned}
A^{\prime}(t & +v)^{-1 / 2}+B(t+v)^{-3 / 2}+o\left(t^{-3 / 2}\right)-A^{\prime} t^{-1 / 2}-B t^{-3 / 2}+o\left(t^{-3 / 2}\right) \\
& =A^{\prime}\left((t+v)^{-1 / 2}-t^{-1 / 2}\right)+B\left((t+v)^{-3 / 2}-t^{-3 / 2}\right)+o\left(t^{-3 / 2}\right) \\
& =\frac{-A^{\prime} v}{2} t^{-3 / 2}+o\left(t^{-3 / 2}\right) .
\end{aligned}
$$


Proof of Theorem 2.1. In the proof we use Lemmas 3.3, 3.4, and 3.5 and the dominated convergence theorem, yielding

$$
\begin{gathered}
-\kappa\left(\theta_{0}\right) \int_{0}^{\infty} \mathrm{e}^{\kappa\left(\theta_{0}\right) v}\left[\mathrm{E}_{(x, z) ; \theta_{0}}\left(\mathrm{e}^{\left(\theta_{0}+\kappa\left(\theta_{0}\right)\right) Z(\tau)} ; \tau>t\right)\right. \\
\left.-\mathrm{E}_{(x, z) ; \theta_{0}}\left(\mathrm{e}^{\left(\theta_{0}+\kappa\left(\theta_{0}\right)\right) Z(\tau)} ; \tau>t+v\right)\right] \mathrm{d} v \\
\sim \frac{H(x-z)(r-1)}{\sqrt{\lambda\left(\theta_{0}\right)}} t^{-3 / 2}\left(-\kappa\left(\theta_{0}\right)\right) \int_{0}^{\infty} v \mathrm{e}^{\kappa\left(\theta_{0}\right) v} \mathrm{~d} v \\
=\frac{H(x-z)(r-1)}{-\kappa\left(\theta_{0}\right) \sqrt{\lambda\left(\theta_{0}\right)}} t^{-3 / 2}
\end{gathered}
$$

in the limit as $t \rightarrow \infty$.

\section{Workload of the M/G/1 queue conditioned to stay positive}

Assume that $T_{0}, T_{1}, T_{2}, \ldots$ are exponentially distributed with parameter $\lambda>0$. In this case $\kappa(s)=\lambda\left(\hat{m}_{B}(s)-1\right)-s$, and throughout this section we assume the light tail case to pertain. We will study the workload process $V(t)$ (or, equivalently, the process $X(t)$ ) for an M/G/1 system conditioned to stay positive. Clearly the process $X(t)$ is Markovian. We denote by $X^{\dagger}(t)$ the process $X(t)$ killed at the exit from $(0, \infty)$; that is,

$$
X^{\dagger}(t)= \begin{cases}X(t), & t<\tau, \\ 0, & t \geq \tau .\end{cases}
$$

Lemma 4.1. The function $h(x)=x \mathrm{e}^{\theta_{0} x}$ is $\gamma$-harmonic for $X^{\dagger}(t)$, where $\gamma=-\kappa\left(\theta_{0}\right)$.

Proof. Note that $X(t)$ is a spectrally positive Lévy process. Using the Wald martingale $\mathcal{E}(t)=\mathrm{e}^{-\theta_{0} x} \mathrm{e}^{\theta_{0} X(t)-\gamma t}$ (see, e.g. Kyprianou and Palmowski (2005)), the function $h(x)=x \mathrm{e}^{\theta_{0} x}$ is $\left(\gamma, \mathrm{P}_{x}\right)$-harmonic for $X^{\dagger}(t)$ if and only if the function $x$ is $\mathrm{P}_{x} ; \theta_{0}$-harmonic for $X^{\dagger}(t)$, which in turn holds if and only if the process $X^{\dagger}(t)$ is a $\mathrm{P}_{x} ; \theta_{0}$-martingale. Note that under $\mathrm{P}_{x ; \theta_{0}}$ the process $X(t)$ has zero drift. Moreover, the dual process, $\hat{X}(t)=-X(t)$, is upward creeping (has no positive jumps). From Theorem 19 of Bertoin (1996) (see also page 191 there) the renewal function, $\hat{\mathcal{V}}(x)$, of $\hat{X}(t)$ is then equal, up to a constant, to $x$. To complete the proof it thus suffices to demonstrate that $\hat{\mathcal{V}}\left(X^{\dagger}(t)\right)=c X^{\dagger}(t)$ is a $\mathrm{P}_{x} ; \theta_{0}$-martingale. This is straightforward from Problem 7 of Bertoin (1996), since

$$
\hat{\hat{X}}(t)=X(t)
$$

and the dual process $\hat{X}(t)$ also has zero drift.

From the above lemma, we may define a process $X^{\uparrow}(t)$, the process $X(t)$ conditioned to stay positive, through an exponential change of measure using the martingale $\mathcal{E}^{\uparrow}(t)=h\left(X^{\dagger}(t)\right)$ with the good function

$$
h(x)= \begin{cases}x \exp \left(\theta_{0} x\right), & x>0, \\ 0, & x=0 .\end{cases}
$$

We denote the resulting twisted probability measure and the corresponding expectation by $\mathrm{P}^{\uparrow}$ and $\mathrm{E}^{\uparrow}$, respectively. The details can be found in Palmowski and Rolski (2004). In the next proposition we identify the parameters of $X^{\uparrow}(t)$. 
Proposition 4.1. An $M / G / 1$ workload process conditioned to stay positive (or, equivalently, process $X(t)$ conditioned to stay positive) is a piecewise-deterministic Markov process with extended generator of the form

$$
\mathcal{A}^{\uparrow} f(x)=-f^{\prime}(x)+\lambda^{\uparrow}(x) \int_{x}^{\infty}(f(y)-f(x)) Q^{\uparrow}(x, \mathrm{~d} y),
$$

where

$$
\begin{aligned}
\lambda^{\uparrow}(x) & =\frac{\lambda \mathrm{e}^{\theta_{0} x}\left[1 / \lambda+x \hat{m}_{B}\left(\theta_{0}\right)\right]}{x \mathrm{e}^{\theta_{0} x}}=\frac{1}{x}+\lambda \hat{m}_{B}\left(\theta_{0}\right), \\
Q^{\uparrow}(x, \mathrm{~d} y) & =\frac{y \mathrm{e}^{\theta_{0} y}}{\mathrm{e}^{\theta_{0} x}\left[1 / \lambda+x \hat{m}_{B}\left(\theta_{0}\right)\right]} F_{B}(\mathrm{~d} y-x) .
\end{aligned}
$$

Proof. Following Theorem 3.1 of Palmowski and Rolski (2004), under the new probability measure the $\mathrm{M} / \mathrm{G} / 1$ workload process is a piecewise-deterministic Markov process with parameters

$$
\lambda^{\uparrow}(x)=\frac{\lambda \bar{G}(x)}{h(x)} \quad \text { and } \quad Q^{\uparrow}(x, \mathrm{~d} y)=\frac{h(y)}{\bar{G}(x)} F_{B}(\mathrm{~d} y-x),
$$

where the normalization function is

$$
\begin{aligned}
\bar{G}(x) & =\int_{x}^{\infty} h(y) F_{B}(\mathrm{~d} y-x) \\
& =\int_{x}^{\infty} y \mathrm{e}^{\theta_{0} y} F_{B}(\mathrm{~d} y-x) \\
& =\int_{0}^{\infty}(y+x) \mathrm{e}^{\theta_{0}(y+x)} F_{B}(\mathrm{~d} y) \\
& =\mathrm{e}^{\theta_{0} x}\left[\int_{0}^{\infty} y \mathrm{e}^{\theta_{0} y} F_{B}(\mathrm{~d} y)+x \int_{0}^{\infty} \mathrm{e}^{\theta_{0} y} F_{B}(\mathrm{~d} y)\right] \\
& =\mathrm{e}^{\theta_{0} x}\left[\hat{m}_{B}^{\prime}\left(\theta_{0}\right)+x \hat{m}_{B}\left(\theta_{0}\right)\right] \\
& =\mathrm{e}^{\theta_{0} x}\left[\frac{1}{\lambda}+x \hat{m}_{B}\left(\theta_{0}\right)\right] .
\end{aligned}
$$

The final equality holds because $\kappa^{\prime}\left(\theta_{0}\right)=0$ implies that $\hat{m}_{B}^{\prime}\left(\theta_{0}\right)=1 / \lambda$. The formulae for $\lambda^{\uparrow}(x)$ and $Q^{\uparrow}(x, \mathrm{~d} y)$ immediately follow.

Note that $X^{\uparrow}(t)$ is transient. Indeed, for $0<a<x<b$, let $\tau_{a}^{-}=\inf \left\{t \geq 0: X^{\uparrow}(t)<a\right\}$ and $\tau_{b}^{+}=\inf \left\{t \geq 0: X^{\uparrow}(t)>b\right\}$. Then $\mathcal{E}^{\uparrow}\left(t \wedge \tau_{a}^{-} \wedge \tau_{b}^{+}\right)$is a uniformly integrable martingale. Hence, using the optional stopping theorem, we have

$$
\mathrm{P}_{x}^{\uparrow}\left(\tau_{a}^{-}<\tau_{b}^{+}\right)=\frac{a}{x} \mathrm{e}^{\theta_{0}(a-x)} \mathrm{P}_{x}\left(\tau_{a}^{-}<\tau_{b}^{+}\right) .
$$

By taking $b \rightarrow \infty$ and keeping in mind that $\mathrm{P}_{x}\left(\tau_{a}^{-}<\infty\right)=1$, we obtain

$$
\mathrm{P}_{x}^{\uparrow}\left(\tau_{a}^{-}<\infty\right)=\frac{a}{x} \mathrm{e}^{\theta_{0}(a-x)}<1 .
$$

From Kyprianou (1971) we know that a quasistationary distribution $\mu$ exists for the M/G/1 queue in which the service time has a meromorphic moment generating function

$$
\hat{m}_{\mu}(t)=\frac{\theta_{0}+\lambda\left(1-\hat{m}_{B}\left(\theta_{0}\right)\right)}{\theta_{0}-t+\lambda\left(\hat{m}_{B}(t)-\hat{m}_{B}\left(\theta_{0}\right)\right)} .
$$


Recently, Kyprianou and Palmowski (2006) proved the existence of a quasistationary distribution for a more general model than that considered here, namely one where $X(t)$ is a Lévy process with light-tailed jumps. Thus, following Proposition 4.1 of Palmowski and Rolski (2004), we know that there exists an invariant measure $\pi$ (which is not finite in this case) for the transition function, $p_{t}^{\uparrow}(x, B)$, of $X^{\uparrow}(t)$. That is, $\pi$ satisfies

$$
\int_{A} \pi(\mathrm{d} x) p_{t}^{\uparrow}(x, B)=\pi(B)
$$

for all bounded Borel subsets $B$. Furthermore, we have $\int_{0}^{\infty}(1 / x) \mathrm{e}^{-s x} \pi(\mathrm{d} x)<\infty$ for $s>0$. Note that it follows, from (4.1), that $\int_{A} \pi(\mathrm{d} x) \mathcal{A}^{\uparrow} f(x)=0$ for all functions $f$ from the domain of $\mathcal{A}^{\uparrow}$ such that $\mathcal{A}^{\uparrow} f$ is $\pi$-integrable. In the following corollary we obtain the Laplace-Stieltjes transform, $\hat{l}_{\pi}(s)$, of the invariant measure $\pi$.

Corollary 4.1. The process $X^{\uparrow}(t)$ has an invariant measure $\pi$ given, up to a multiplicative constant, by its Laplace transform,

$$
\hat{l}_{\pi}(s)=\frac{\left(\theta_{0}+\lambda\left(1-\hat{m}_{B}\left(\theta_{0}\right)\right)\right)\left(\lambda \hat{m}_{B}^{\prime}\left(\theta_{0}-s\right)-1\right)}{\left(s+\lambda\left(\hat{m}_{B}\left(\theta_{0}-s\right)-\hat{m}_{B}\left(\theta_{0}\right)\right)\right)^{2}}, \quad s>0 .
$$

Proof. For $f(x)=\mathrm{e}^{-s x}, x>0, s>0$, we have

$$
\left(\mathcal{A}^{\uparrow} f\right)(x)=s \mathrm{e}^{-s x}+\lambda \int_{0}^{\infty}\left(\mathrm{e}^{\left(\theta_{0}-s\right) y}-\mathrm{e}^{\theta_{0} y}\right) \frac{y+x}{x} \mathrm{e}^{-s x} F_{B}(\mathrm{~d} y) .
$$

We now integrate both sides with respect to $\pi(\mathrm{d} x)$, to obtain

$$
\begin{aligned}
\int_{0}^{\infty}\left(\mathcal{A}^{\uparrow} f\right)(x) \pi(\mathrm{d} x)= & s \hat{l}_{\pi}(s)+\lambda \int_{0}^{\infty} y\left(\mathrm{e}^{\left(\theta_{0}-s\right) y}-\mathrm{e}^{\theta_{0} y}\right) F_{B}(\mathrm{~d} y) \int_{0}^{\infty} \frac{1}{x} \mathrm{e}^{-s x} \pi(\mathrm{d} x) \\
& +\lambda \int_{0}^{\infty}\left(\mathrm{e}^{\left(\theta_{0}-s\right) y}-\mathrm{e}^{\theta_{0} y}\right) F_{B}(\mathrm{~d} y) \hat{l}_{\pi}(s) .
\end{aligned}
$$

If $\pi$ is an invariant measure then $\int_{0}^{\infty}\left(\mathcal{A}^{\uparrow} f\right)(x) \pi(\mathrm{d} x)=0$ (see Davis (1993, Theorem 34.19)). Hence,

$$
s \hat{l}_{\pi}(s)+\lambda\left(\hat{m}_{B}^{\prime}\left(\theta_{0}-s\right)-\hat{m}_{B}^{\prime}\left(\theta_{0}\right)\right) \int_{0}^{\infty} \frac{1}{x} \mathrm{e}^{-s x} \pi(\mathrm{d} x)+\lambda\left(\hat{m}_{B}\left(\theta_{0}-s\right)-\hat{m}_{B}\left(\theta_{0}\right)\right) \hat{l}_{\pi}(s)=0
$$

for $s>0$. Now, using the fact that

$$
\int_{0}^{\infty} \frac{1}{x} \mathrm{e}^{-s x} \pi(\mathrm{d} x)=\int_{s}^{\theta_{0}} \hat{l}_{\pi}(t) \mathrm{d} t+\int_{0}^{\infty} \frac{1}{x} \mathrm{e}^{-\theta_{0} x} \pi(\mathrm{d} x)
$$

which is finite, we have

$$
\frac{-\hat{l}_{\pi}(s)}{\int_{s}^{\theta_{0}} \hat{l}_{\pi}(t) \mathrm{d} t+\int_{0}^{\infty}(1 / x) \mathrm{e}^{-\theta_{0} x} \pi(\mathrm{d} x)}=-\frac{1-\lambda \hat{m}_{B}^{\prime}\left(\theta_{0}-s\right)}{s+\lambda\left(\hat{m}\left(\theta_{0}-s\right)-\hat{m}_{B}\left(\theta_{0}\right)\right)} .
$$

Hence, we obtain

$$
\int_{s}^{\theta_{0}} \hat{l}_{\pi}(t) \mathrm{d} t+\int_{0}^{\infty} \frac{1}{x} \mathrm{e}^{-\theta_{0} x} \pi(\mathrm{d} x)=\frac{c}{s+\lambda\left(\hat{m}_{B}\left(\theta_{0}-s\right)-\hat{m}_{B}\left(\theta_{0}\right)\right)}
$$


for some constant $c$. By choosing the multiplicative constant in $\pi$ so that

$$
\int_{0}^{\infty} \frac{1}{x} \mathrm{e}^{-\theta_{0} x} \pi(\mathrm{d} x)=1,
$$

we can show that $c=\theta_{0}-\lambda \hat{m}_{B}\left(\theta_{0}\right)+\lambda$ and, thus, that

$$
\int_{t}^{\theta_{0}} \hat{l}_{\pi}(s) \mathrm{d} s=\frac{\theta_{0}-\lambda \hat{m}_{B}\left(\theta_{0}\right)+\lambda}{t+\lambda\left(\hat{m}_{B}\left(\theta_{0}-t\right)-\hat{m}_{B}\left(\theta_{0}\right)\right)}-1 .
$$

Differentiation completes the proof.

\section{Acknowledgements}

The authors are grateful to Søren Asmussen for pointing out Cox and Smith (1961). Thanks are also due to the anonymous referee who uncovered a gap in the arguments in a previous version of the paper. Part of this collaborative work was carried out when the first author was a researcher at EURANDOM and the second author was visiting EURANDOM. Both authors would like to express their respective thanks to this institution for its hospitality and support. In addition, the first author acknowledges support through KBN grant 1 P03A 03128 and NWO grant 613.000.310. The second author acknowledges support through KBN grant 2 P03A 02023.

\section{References}

Asmussen, S. (2003). Applied Probability and Queues, 2nd edn. Springer, New York.

Bertoin, J. (1996). Lévy Processes (Camb. Tracts Math. 121). Cambridge University Press.

Bertoin, J. And Doney, R. A. (1994). On conditioning a random walk to stay nonnegative. Ann. Prob. 22, $2152-2167$.

Chover, J., Ney, P. And Wainger, S. (1973). Functions of probability measures. J. Anal. Math. 26, $255-302$.

Cox, D. R. AND Smith, W. L. (1961). Queues. Methuen, London.

Davis, M. H. A. (1993). Markov Models and Optimization (Monogr. Statist. Appl. Prob. 49). Chapman and Hall, London.

Doney, R. A. (1989). On the asymptotic behaviour of first passage times for transient random walk. Prob. Theory Relat. Fields 81, 239-246.

Feller, W. (1966). An Introduction to Probability Theory and Its Applications, Vol. II. John Wiley, New York.

Iglehart, D. L. (1974). Random walks with negative drift conditioned to stay positive. J. Appl. Prob. 11, $742-751$.

Keener, R. W. (1992). Limit theorems for random walks conditioned to stay positive. Ann. Prob. 20, 801-824.

Knight, F. B. (1969). Brownian local times and taboo processes. Trans. Amer. Math. Soc. 143, 173-185.

Kyprianou, A. E. And Palmowski, Z. (2005). A martingale review of some fluctuation theory for spectrally negative Lévy processes. In 38th Seminar on Probability, eds M. Émery et al., Springer, Berlin, pp. 16-29.

Kyprianou, A. E. AND PALMOWski, Z. (2006). Quasi-stationary distributions for Lévy processes. To appear in Bernoulli.

KyPRIANOU, E. K. (1971). On the quasi-stationary distribution of the virtual waiting time in queues with Poisson arrivals. J. Appl. Prob. 8, 494-507.

MANDJES, M. AND ZwART, B. (2006). Large deviations of sojourn times in processor sharing queues. Queueing Systems 52, 237-250.

PALMOWSKI, Z. AND Rolski, T. (2002). A technique for exponential change of measure for Markov processes. Bernoulli 8, 767-785.

PAlmowski, Z. ANd Rolski, T. (2004). Markov processes conditioned to never exit a subspace of the state space. Prob. Math. Statist. 24, 339-353.

Petrov, V. V. (1965). On the probabilities of large deviations for sums of independent random variables. Theory Prob. Appl. 10, 287-298.

Petrov, V. V. (1972). Summy Nezavisimyk Slucainyh Velicin. Nauka, Moscow.

Rolski, T., Schmidli, H., Schmidt, V. And Teugels, J. (1999). Stochastic Processes for Insurance and Finance. John Wiley, Chichester. 